\title{
FRONTERAS EN MOVIMIENTO: ¿HACIA DÓNDE VA LA SEGURIDAD CUANDO LA SOBERANÍA MIGRA?
}

\author{
Borders on the Move: where does security go \\ when sovereignty migrates?
}

Elspeth Guild*

\begin{abstract}
Resumen. Este artículo interroga la relación de las fronteras inestables como en el caso de la Unión Europea (UE) - con la seguridad. La Unión Europea (UE) ha lidiado con la cuestión del movimiento de personas, la seguridad y el papel del control fronterizo desde que los controles de las fronteras interiores de los Estados miembros de la UE fueron abolidos en 1995. La presión de los ministerios del Interior de los Estados miembros para percibir el control fronterizo de las personas como fundamento de la seguridad nacional se ha transferido de las fronteras interiores de los Estados miembros a la frontera exterior de la UE. Pero al hacerlo, ha dejado de ser una parte inherente de la soberanía nacional y se ha convertido más bien en un nuevo marco de soberanía que se incorpora a lo que ha sido una organización internacional: la Unión Europea.
\end{abstract}

Palabras clave: seguridad, control fronterizo, soberanía, Union Europea.

\begin{abstract}
This article examines the relationship of unstable borders, such as in the European Union and security. The European Union (EU) has struggled with the issue of movement of persons, security and the role of border control in this regard since the intra-EU Member State border controls on persons were abolished in 1995. The pressure from interior ministries of the Member States to perceive border control on persons as a foundation of national security has been transferred from intra-EU Member State borders to the external border of the EU. But in doing so it is not longer an inherent part of national sovereignty but rather part of a new framework of sovereignty which is incorporated into what has been an international organisation - the European Union.
\end{abstract}

Keywords: security, border control, sovereignty, European Union.

* Profesora Jean Monnet de la Universidad de Radboud, Nijmegen, Holanda, y de Queen Mary, Universidad de Londres. Londres, UK. 
Este artículo interroga la relación de las fronteras inestables -como en el caso de la Unión Europea (UE) - con la seguridad. Las afirmaciones acerca de la soberanía del estado se basan en conceptos de fronteras y en la certeza sobre sus posiciones como marcadores de las exigencias de seguridad. La frontera como una línea que divide los diferentes territorios de soberanía es uno de los asuntos más preciados en las relaciones internacionales. Siempre que exista una frontera entre los estados, entonces es posible pensar la migración - las personas pertenecen a un lado o al otro de la frontera. Si la comunidad internacional se caracteriza por la anarquía, entonces la frontera proporciona una línea de resistencia contra tal desorden. Dentro del estado existe un orden rodeado por la frontera de la soberanía. ¿Qué ocurre, sin embargo, cuando las fronteras comienzan a moverse o reconfigurarse? Si la soberanía está enraizada en la capacidad de un estado para gobernar y ser gobernado -para regular las actividades dentro de su área, sea coercitiva con respecto a la ley penal o distributiva en materia de seguridad social- los contornos de su autoridad son importantes.

¿Cómo cambian las fronteras y su seguridad en tanto lugares donde se efectúan los controles sobre las personas con el propósito de proporcionar seguridad? ¿En qué medida la forma de entender la soberanía del siglo XIX como investida en la solidaridad basada en la nacionalidad circunscrita por fronteras y actuada a través del espectáculo del control fronterizo de personas se vuelve cada vez menos satisfactoria como mecanismo para entender la forma en que la política, la comunidad y la economía son construidas? Cuando la frontera cambia su naturaleza como resultado de las variables concepciones de la soberanía, el propio concepto de migración cambia de una manera fundamental. Sin fronteras no puede haber migración internacional. En lugar de una visión estadocéntrica sobre el movimiento de personas, el estado desaparece y el objeto temático de los estudios migratorios debe ser revisado. Cuando la frontera permanece en pie para algunos propósitos pero no para otros (por ejemplo, el control de la circulación de las personas), la ilusión de los estudios migratorios se mantiene pero las prácticas se transforman. La capacidad de incluir o rechazar a las personas se aleja de su marco más brutal -en el borde del territorio- y, si se ha de ejercer, debe encontrar otros lugares.

\section{¿Comprendiendo la soberanía?}

La estatalidad, de acuerdo con la teoría política occidental, depende de tres elementos centrales: un pueblo, un territorio y una burocracia ${ }^{1}$. El territorio está delimitado por una frontera que mantiene en su lugar a las

\footnotetext{
1 WEBER, Max. The Fundamental Concepts of Sociology.
} 
personas, identificadas como ciudadanos, y la burocracia cuyas acciones están definidas por esas fronteras. Una condición necesaria de la estatalidad es que la burocracia haya establecido una pretensión sostenible al monopolio del uso legítimo de la violencia dentro del territorio. El término "autoridad" se utiliza a menudo en lugar de "violencia", pero el concepto básico del estado es su capacidad para determinar y hacer cumplir su orden que, de otra manera, se llama "soberanía".

Las relaciones entre estados giran en torno a los dilemas que se plantean en el contexto de estas características fijas de las personas, las fronteras y el orden $^{2}$. La Unión Europea, sin embargo, desafía cada vez más este acuerdo teórico. A continuación, examino la deconstrucción de los controles fronterizos de personas en la UE. Al hacer esto, busco tratar dos cuestiones: 1. Qué ocurre con la relación entre migración y seguridad: ¿dónde se establece si ya no en las fronteras soberanas?; 2. La soberanía se establece, hasta ahora, en los controles fronterizos como el lugar en el cual es puesta en juego más emblemáticamente, entonces ìhacia dónde se dirige cuando migra lejos de la frontera?

En la UE, las personas ya no pertenecen exclusivamente a los estados soberanos que la conforman (28 en el último recuento), sino que han adquirido un estatus -ciudadanía de la Unión Europea- que los vuelve sujetos de un actor no estatal. Este actor, la UE, es capaz de conferirles derechos que un estado, ya sea el de su nacionalidad o de su residencia (es decir, un estado anfitrión en lo que respecta a un migrante), no puede (o, como en el caso de la reagrupación familiar, no desea) otorgar ${ }^{3}$. Lo más importante, para mis propósitos, es que el símbolo clásico de la soberanía estatal -el control fronterizo sobre las personas que entran y salen del estado, lo cual proporciona el mecanismo para que el estado evidencie su soberanía a sus ciudadanos y a los extranjeros- ya no es posible para (casi todos) los Estados miembros de la UE.

\section{¿Dónde está el territorio?}

El territorio sobre el cual el estado ejerce su jurisdicción es desafiado por la UE. Mientras que el estado soberano europeo sigue siendo la piedra angular de la UE, el símbolo del territorio -la frontera del estado- sólo es visible en lugares muy específicos en la mayor parte de la UE. Esta frontera invisible es conocida comúnmente como la frontera de Schengen, tras el acuerdo firmado en 1985 entre cinco Estados miembros, donde adelantándose a la legislación de la UE, acordaron abolir tanto los controles fronterizos intraestatales sobre el movimiento de mercancías y personas como los controles comunes sobre la

\footnotetext{
ALBERT, Mathias, JACOBSON, David, LAPID, Yosef. Identities, Borders, Orders: Rethinking International Relations Theory.

3 BALDACCINI, Anneliese, GUILD, Elspeth, TONER, Helen. Whose Freedom, Security and Justice?: EU Immigration and Asylum Law and Policy.
} 
admisión de no nacionales de la UE que arriban al espacio común ${ }^{4}$. Poco a poco, todos los demás Estados miembros suscribieron el acuerdo con la excepción de Irlanda y el Reino Unido. El primer acuerdo fue complementado con un segundo acuerdo en 1990 y la supresión de los controles fronterizos comunes sobre la circulación de personas tuvo lugar recién el 25 de marzo de 1995: Bélgica, Francia (con algunos retrasos debido a la política de drogas holandesa), Alemania, Luxemburgo, los Países Bajos, Portugal y España suprimieron los controles en esa fecha; les siguieron Italia y Austria en 1997, Grecia en 2000 y Dinamarca, Suecia, Finlandia, Noruega e Islandia en 2001 -aunque los dos últimos no son Estados miembros de la UE- y los Estados miembros de Europa central, incluyendo Suiza -otro estado fuera de la UE- en $2008^{5}$. El resultado fue la transformación de lo que antes habían sido las fronteras sujetas al control del movimiento de personas en fronteras interiores sin controles (por ejemplo, aquella entre Polonia y Alemania) y la creación de nuevas fronteras exteriores para la zona común a los efectos de controlar a las personas (por ejemplo, Polonia y Rusia) $)^{6}$.

El control de las personas en las fronteras comunes de los estados de la UE ya no es admisible como resultado de la legislación de la $\mathrm{UE}^{7}$. Incluye unos 500 millones de ciudadanos de la Unión Europea ${ }^{8}$. Las sorpresas de la UE son Irlanda y el Reino Unido por elección, Bulgaria y Rumanía, que aún no han sido admitidos, y Chipre que, como una isla con un territorio en disputa, no es capaz de participar. Dinamarca participa a través de un acuerdo intergubernamental con 23 países miembros. Islandia, Noruega y Suiza que no son Estados miembros de la UE -como Dinamarca- también participan en la frontera invisible de Schengen a través de un acuerdo. La entrada de Suiza no marcó la estabilización del territorio de Schengen. Bulgaria, Chipre y Rumanía han de entrar en el sistema de Schengen tan pronto como sus sistemas sean considerados lo suficientemente seguros.

Como la UE no ha terminado de ampliarse, es probable que el área Schengen, que la imita pero que no es idéntica a ella, también se agrande. Mientras que a Irlanda y el Reino Unido se les ha permitido mantener sus controles fronterizos nacionales con los demás Estados miembros de la UE

\footnotetext{
${ }^{4}$ BROUWER, Evelien. Digital Borders and Real Rights: Effective Remedies for Third-Country Nationals in the Schengen Information System.

5 ATGER, Anaïs Faure. The Abolition of Internal Border Checks in an Enlarged Schengen Area: Freedom of movement or a scattered web of security checks?

6 POTEMKINA, Olga, KAVESHNIKOV, Nikolay. Russia and the European Union: 'Cold Summer'. 7 Al momento de escribir este artículo (2015) hay seis casos atípicos que no están incluidos en el espacio sin fronteras interiores: por elección, Irlanda y el Reino Unido; y por medio de la exclusión temporal, Bulgaria, Croacia, Chipre y Rumanía.

8 BALZACQ, Thierry, CARRERA, Sergio. Migration, Borders and Asylum: Trends and Vulnerabilities in EU Policy.
} 
(incluidos en el Tratado de Lisboa como protocolos), todos los estados que se unen a la UE son obligados a participar plenamente en el proyecto Schengen. Los estados candidatos actuales de la UE son Islandia, la ex República Yugoslava de Macedonia, Montenegro, Serbia y Turquía. Un potencial país candidato es Kosovo, al amparo de la Resolución 1244 del Consejo de Seguridad de las Naciones Unidas ${ }^{9}$.

La forma en que se produce el movimiento de personas -sean ciudadanos de la UE o nacionales de terceros países- a través de las fronteras nacionales de los estados participantes de Schengen es incontrolable. El objetivo (y la realidad) es que sea similar al movimiento de personas dentro del territorio de un estado ${ }^{10}$. La autoridad de la soberanía que se expresa en los controles fronterizos está excluida en el régimen interno de Schengen. En cuanto a los controles de inmigración de personas que intentan entrar en el territorio Schengen desde el exterior, se aplica un conjunto común de controles dado que la soberanía de todos los estados participantes es inherente al control externo que tiene lugar en cualquiera de ellos. Mientras el régimen de Schengen fue intergubernamental, regulado por un acuerdo internacional, la naturaleza fundamental del control no cambió - una expresión de soberanía. Sin embargo, una vez que el régimen se transformó en legislación de la UE, su naturaleza se convirtió en uno gobernado por la jerarquía, lo cual es central para el derecho comunitario. La capacidad del estado para imponer un orden es modificada y condicionada en la medida en que ese estado está regido por el principio del estado de derecho.

\section{La soberanía, el derecho y las personas en las fronteras}

El ejercicio de la capacidad del estado para determinar su orden respecto a sus fronteras se encuentra en sus leyes y reglamentos sobre la inmigración y los controles fronterizos. En esta sección examinaré el cambio en la ley y la capacidad legislativa del estado como resultado de ingresar en el régimen de Schengen.

Si bien el primer Acuerdo de Schengen data de 1985 y establece el objetivo común de suprimir los controles fronterizos entre los cinco estados que lo firmaron, éste no tuvo lugar en el vacío. De hecho, se consolidó como un acuerdo controlado por los socios justo antes de que la UE tratara de dominar el campo. En 1986, la UE (bajo el nombre de Comunidad Económica Europea en aquél momento) acordó el Acta Única Europea, la

9 Cf. <http://ec.europa.eu/enlargement/potential-candidate-countries/index_en.htm>.Consultado: 25.06.2015.

${ }^{10}$ Mientras se excluyen los controles migratorios, los controles de policía no lo son. Sin embargo, una característica clave de los controles policiales, en comparación con los controles migratorios, es que normalmente en las democracias liberales deben estar motivados. 
primera modificación importante al tratado que incluía el deber de abolir los controles fronterizos sobre la circulación de personas a través de las fronteras de los Estados miembros para el 31 de diciembre de 1992, pero sin hacer ninguna mención a la frontera exterior ${ }^{11}$. Esto fue parte del gran proyecto de 1992 para completar el mercado interior de la UE, llevado a cabo por el entonces presidente de la Comisión, Jacques Delors. Es difícil pasar por alto la superposición obvia (para evitar llamarla competencia) entre el Acuerdo de Schengen y el Acta Única Europea. No obstante, las instituciones de la UE y los Estados Schengen decidieron proceder a través de dos universos paralelos en cuanto a la supresión de los controles fronterizos en lugar de hacer frente a esa incoherencia bastante obvia ${ }^{12}$. Las razones son múltiples, pero dos tienen una resonancia particular: en primer lugar, el Reino Unido, bajo la primera ministra Margaret Thatcher, se opuso implacablemente a la supresión de los controles fronterizos sobre las personas con los demás Estados miembros (esta posición ha sido seguida hasta ahora por todos los siguientes primer ministros británicos); en segundo lugar, la posibilidad de que las normas fronterizas se conviertan en una legislación comunitaria vinculante antes que en un ejercicio de la soberanía del estado, aún cuando dicha soberanía debiera ser ejercida temporalmente para abolir su existencia, era un paso que iba demasiado lejos y demasiado rápido ${ }^{13}$.

Esta disposición era, por supuesto, insostenible en el largo plazo, el cual llegó bastante rápido. Con la ampliación continua de la participación en el proyecto Schengen y el creciente nivel de detalle que el sistema Schengen producía sobre cómo no se controlarían las fronteras entre los Estados miembros, cómo se controlarían los estados extra-comunitarios, cómo se emitirían las visas, qué excepciones deberían existir y cómo deberían ser aplicadas, la administración y la aplicación coherente del sistema se hicieron cada vez más frágiles. No existía ningún mecanismo policial eficaz para asegurarse de que los estados participantes hicieran lo que se suponía que debían hacer. Al final, los Estados Schengen tiraron la toalla y le entregaron todo el acuerdo -a esa altura bastante desordenado- a la UE en virtud de un protocolo del Tratado de Amsterdam (que tan pronto como entró en vigor transfirió el protocolo a los tratados de la CE y de la UE). Todo esto tuvo lugar en 1999, pero en 2008 la UE aún no había conseguido integrar plenamente el legado de Schengen.

Bajo los acuerdos de Schengen transpuestos a la legislación de la UE, se aplica un conjunto común de reglas que el estado ya no tiene la facultad exclusiva de modificar o cambiar en tanto ejercicio de soberanía

\footnotetext{
${ }^{11}$ GUILD, Elspeth. Moving the Borders of Europe.

${ }^{12}$ ELSEN, Charles. Schengen et la cooperation dans les domains de la justice et des affaires interiors. Besoins actuels et options futures.

${ }^{13}$ GORTÁZAR, Cristina. Spain: Two Immigration Acts at the End of the Millennium.
} 
sobre el movimiento de personas hacia y desde el estado ${ }^{14}$. De hecho, la administración que lleva a cabo el control del territorio está cada vez más colonizada por normas de la UE que el funcionario debe garantizar que se Ileven a cabo de forma adecuada en detrimento de las leyes fronterizas del estado soberano. En el caso de un conflicto entre la normativa nacional y la de la UE respecto de las fronteras, el funcionario está obligado a aplicar la legislación de la UE y no debe tener en cuenta la legislación nacional que sea contraria. Este principio está en el centro del sistema de la UE y, como tal, se aplica igualmente a las normas sobre circulación tanto de las personas como de los bienes, servicios o capital ${ }^{15}$.

El Código de Fronteras de Schengen entró en vigor el 13 de octubre de 2006. Proporciona la ley común de la UE sobre el modo en que las fronteras interiores y exteriores deben ser gestionadas en el ámbito de la circulación de las personas. En tanto Reglamento (562/2006), el Código tiene efecto directo en los Estados miembros y no es objeto de transposición a la legislación nacional. La abolición de los controles en las fronteras interiores es especificada en el Reglamento y, por lo tanto, ya no es competencia de la legislación nacional. Del mismo modo, los deberes de los guardias de fronteras de admitir o denegar la entrada a nacionales de terceros países (aunque limitada principalmente a los que llegan para estancias cortas) en la frontera exterior, se establecen ahora en el Código, que es la ley de la UE. Por lo tanto, la UE ha adquirido competencias de control fronterizo interno y externo común, a pesar de que no es un estado.

En relación a las fronteras interiores de los Estados miembros de Schengen, entre los estados que participan en el sistema, todas las personas, ya sean ciudadanos de un Estado miembro o nacionales de terceros países, tienen derecho a circular libremente en todo el territorio durante un máximo de tres meses (solo aquellos que buscan protección internacional o están presentes irregularmente están excluidos de este derecho). Este es un ejemplo notable de eliminación de obstáculos de seguridad para el disfrute de la libertad -de hecho la más básica de las libertades-, la libertad de movimiento. El libre movimiento de personas en la UE es de inmediato uno de los derechos más visibles y de gran valor para las personas que viven en la UE. Incluye el derecho de las personas a moverse, debido a una amplia variedad de razones, a través de la UE y se ha ampliado gradualmente para incluir a los nacionales de terceros países. Hay que reconocer, no obstante, que muy a menudo hay una brecha entre los derechos de los individuos y su ejercicio, primero a manos de las administraciones de los Estados miembros distintos al de su nacionalidad y, segundo, por los actores

\footnotetext{
${ }^{14}$ GUILD, Elspeth. The Uses and Abuses of Counter-Terrorism Policies in Europe: The Case of the 'Terrorist Lists'.

15 TRIDIMAS, Takis. The General Principles of EU Law.
} 
de los sectores económicos y culturales ${ }^{16}$. Todavía para muchas personas que tienen derecho a la libre circulación, la recompensa personal más evidente de la legislación de la UE ha sido el derecho de no toparse, en absoluto, con la administración pública. La frontera interior se caracteriza principalmente por la ausencia de controles sobre las personas.

Los Estados miembros, no obstante, de acuerdo con la legislación de la UE, tienen el derecho a saber quién está en su territorio. El derecho a la libre circulación no es absoluto. Los intereses de la política pública, la seguridad pública y la salud pública son reconocidos expresamente en la legislación de la UE como los motivos por los que el derecho a la libre circulación de las personas podría ser limitado. Sin embargo, en el ejercicio de sus funciones para garantizar la política pública, la seguridad y la salud, los Estados miembros deben respetar la prioridad del derecho a la libre circulación. Las excepciones son exactamente esas. Su uso debe ser justificado por los Estados miembros cuando quieran beneficiarse de ellas y las medidas adoptadas deben ser proporcionales al interés legítimo a ser protegido. Tal como declaró el Tribunal de Justicia, "[e]l requisito de seguridad jurídica significa que la situación jurídica resultante de la implementación de medidas nacionales debe ser lo suficientemente clara y precisa para permitir a los individuos afectados conocer el alcance de sus derechos y obligaciones" ${ }^{\prime 17}$. No obstante, sin la facultad de mantener a los funcionarios en las fronteras interiores de los Estados miembros para controlar a las personas, no hay manera de que el Estado miembro individual pueda utilizar el mecanismo de las fronteras para este fin. El derecho del estado de saber quién está en su territorio ya no está relacionado con la frontera, sino con la organización del estado - por ejemplo, los registros de población, etc.

En cuanto a las fronteras exteriores de la UE, iqué tipo de fronteras son de acuerdo con sus normas? El Código especifica que "fronteras exteriores" son las fronteras terrestres de los Estados miembros, incluidas las fronteras fluviales, lacustres y marítimas, los aeropuertos, y los puertos fluviales, marítimos y lacustres, siempre que no sean fronteras interiores (artículo 2 (2)). Además, aquellas fronteras son las que “...sólo podrán cruzarse por los pasos fronterizos y durante las horas de apertura establecidas. Las horas de apertura estarán indicadas claramente en todo paso fronterizo que no esté abierto las 24 horas del día". Así, la frontera exterior, para el propósito de individuos que buscan cruzarla, consiste en una serie de puntos definidos tanto en el espacio como en el tiempo. Según la Comisión Europea, en la región hay 300 millones de cruces fronterizos externos al año. De acuerdo con los Estados miembros,

\footnotetext{
${ }^{16}$ CARBY-HALL, Jo. The Treatment of Polish and A8 Economic Migrants in the European Union Member States.

${ }^{17}$ C-136/03 Dörr y Ünal 2, junio de 2005, párrafo 52.
} 
hay cerca de 900 millones de cruces fronterizos externos. Ambos conjuntos de datos incluyen ciudadanos de la UE y de terceros países ${ }^{18}$. Para el Consejo, la frontera exterior tiene alrededor de 3,6 millones de kilómetros de largo. No está claro por qué hay una discrepancia importante entre la Comisión Europea y los Estados miembros en relación con el número de cruces fronterizos externos. Sin embargo, la respuesta puede ser que, si bien la frontera exterior de la UE es "pixelada", las fronteras exteriores de los Estados miembros sigan siendo percibidas como "líneas" en el mapa.

A los Estados miembros se les solicitó que notificaran a la Comisión Europea dónde se encuentran los puntos que constituyen la frontera exterior para la circulación de personas. Hay 1.792 pasos fronterizos exteriores designados con controles en la UE. De éstos, 665 son fronteras aéreas, 871 son fronteras marítimas y sólo 246 son fronteras terrestres ${ }^{19}$. Sobre la base de esta información, uno podría ser perdonado por pensar que la UE es una isla al igual que Australia, más bien lejos de todos lados y, por lo tanto, alentando viajes por mar y aire. Como cualquier mirada casual a un mapa revelará, esto no es del todo correcto geográficamente, por lo que la explicación al respecto debe estar en otra parte. Es evidente que entre los Estados miembros hay una marcada preferencia por designar pasos fronterizos exteriores con controles en los puertos marítimos de la UE. Esto puede deberse a que la infraestructura para llevar a cabo un control en los puertos marítimos está bien desarrollada. El segundo lugar más popular para el cruce de fronteras exteriores con controles son los aeropuertos. A los efectos del control, los aeropuertos son muy convenientes ya que reúnen a muchos viajeros a la vez y los conducen a través de diversos controles en una sucesión rápida. Las fronteras menos populares son las terrestres. Sólo 236 lugares han sido designados como pasos fronterizos exteriores terrestres con controles ${ }^{20}$. Sin duda, la longitud de las fronteras terrestres de la UE con sus vecinos hace que la administración sea difícil desde el punto de vista de los pasos fronterizos designados con controles.

El Código ha cambiado la naturaleza de la frontera exterior de los Estados miembros como una cuestión de derecho. El acto del cruce individual de una frontera exterior, que está destinado a ser capturado en el Código, no parece corresponder a un entendimiento común. El conjunto de individuos que entra en la UE de acuerdo con la Comisión y de acuerdo con los Estados miembros varía en un factor de 1:3. Quizás sea ésta la expresión matemática de la soberanía.

\footnotetext{
${ }^{18}$ Comisión Europea, Comunicación sobre los Próximos Pasos en la Gestión de Fronteras en la Unión Europea COM (2008) 69 final.

${ }^{19}$ Artículo 34 del Código de Fronteras de Schengen.

${ }^{20}$ Comisión Europea, Comunicación sobre los Próximos Pasos en la Gestión de Fronteras en la Unión Europea COM (2008) 69 final.
} 


\section{¿Soberanía más allá de la frontera?}

La frontera de la UE divide a la población mundial en dos grandes grupos (trataré la cuestión de las divisiones más adelante). Como he indicado anteriormente, hay un grupo de personas que ya están dentro de la frontera exterior. Este grupo puede ser fragmentado en muchos subgrupos, por ejemplo, nacionales de la UE, nacionales de terceros países con estatus de residencia de largo plazo en la UE, refugiados, personas presentes de manera irregular, etc. Por el momento, las dejaré de lado y consideraré aquella parte de la población mundial que no está dentro de la UE. Esta gran mayoría de personas, de acuerdo con la UE a los efectos de su gestión de las fronteras, se puede subdividir en dos grupos principales, los cuales están sujetos a una mayor fragmentación. El primer grupo clave remite a aquellos que necesitan visa para entrar en la UE por una estancia de tres meses y a aquellos que no la requieren.

El Reglamento de Visas (539/2001 en su versión modificada) establece un listado de países cuyos ciudadanos necesitan visas para entrar en la UE y aquellos que no ${ }^{21}$. Los nacionales de países que no requieren una visa para ingresar a la UE pueden llegar a la frontera exterior de la UE y deben ser admitidos o rechazados de acuerdo con el Código. Esto ocurre porque al no estar incluidos sus países en el Reglamento de Visas, la directiva de la UE sobre sanciones a los transportistas (2001/51) no dará lugar a una multa a cualquier transporte que los traigan a la UE. Los países que no están en la lista negra ascienden a 44 (aunque seis fueron añadidos en 2008) e incluyen América del Norte y la mayor parte de América del Sur -con algunas excepciones-, Australia, Japón y una pequeña cantidad de países -bastante ricos- del sudeste Asiático. Hay más de 120 países y territorios en la lista de negra de visas. Estos países incluyen a casi todos los de África, Oriente Medio -excepto Israel-, países europeos no-miembros de la UE, Asia y el subcontinente indio. La división de los países en dos listas blanca y negra- refleja el origen étnico, la religión y el PIB del mundo ${ }^{22}$. Para los nacionales de estos países, la obtención de una visa es un requisito previo necesario para viajar a la UE. En 2014 se solicitaron poco menos de 17 millones de visas Schengen de corta duración y fueron emitidas 15,5 millones de visas. La tasa de rechazo fue del $5,1 \%^{23}$. Sin embargo, el porcentaje por país varía radicalmente. Por ejemplo, el 26,4\% de las solicitudes rechazadas son de Costa de Marfil, pero sólo el 3\% son de China $^{24}$.

\footnotetext{
${ }^{21}$ MELONI, Annalisa. Visa Policy Within the European Union Structure.

${ }^{22}$ BIGO, Didier, GUILD, Elspeth (eds.). Controlling Frontiers: Free Movement Into and Within Europe.

${ }^{23}$ Cf. <http://ec.europa.eu/dgs/home-affairs/what-we-do/policies/borders-and-visas/visa-policy/ index_en.htm>. Consulado el 25 de junio de 2015.

${ }^{24}$ Cf. $\quad<$ http://ec.europa.eu/dgs/home-affairs/what-we-do/policies/borders-and-visas/visa-policy/ index_en.htm>. Consulado el 25 de junio de 2015.
} 
Una vez que un individuo tiene una visa Schengen válidamente emitida, él o ella tiene una presunción de admisión en el territorio de Schengen, independientemente de dónde él o ella llegue primero. De este modo, un individuo de Costa de Marfil, que obtuvo una visa Schengen de las autoridades francesas puede esperar ser admitido si él o ella llega a París, Frankfurt o Estocolmo - la compañía no tiene miedo a una multa por llevar a ese individuo a la UE y cuando él o ella llega, se aplica el Código. El artículo 5 del Código requiere que la persona muestre una cantidad de elementos y proporcione información para ser admitido en la frontera exterior: un documento de viaje válido y una visa (en caso que sea solicitada); justificar el propósito y condiciones de la estancia prevista (aunque una lista de los documentos de apoyo se incluye en un anexo) y mostrar fondos suficientes para la subsistencia, los cuales son establecidos como una suma fija diaria, aunque las cantidades varían ampliamente entre los Estados miembros; no estar notificado como una persona a ser excluida de la UE (es decir, en base al Sistema de Información de Schengen); y no ser una amenaza para la política pública, la seguridad nacional, la salud pública o las relaciones internacionales de alguno de los Estados miembros.

Si bien esto puede parecer una lista bastante considerable y laxa, en la práctica, el funcionario de inmigración en el puerto de entrada, sobre todo si se trata de un aeropuerto, tendrá normalmente menos de un minuto para comprobar los documentos e identidad antes de tomar la decisión. Cualquier período más largo de tiempo para considerar al individuo puede dar lugar a grandes embotellamientos de tráfico. Además, si el funcionario niega la admisión del individuo, él o ella tiene derecho a recibir las razones por escrito y el derecho de apelación, nuevamente notificado por escrito, contra la denegación (artículo 13). Preparar esto lleva aún más tiempo al funcionario.

En consecuencia, el marcado control del extranjero en lo que hace a la llegada a la UE tiene lugar primero con la decisión de que su país esté o no en la lista negra de visas, lo cual hace que el principal control sea ejercido fuera de la $U E$, cuando el individuo aún se encuentra dentro de su estado de nacionalidad. El control funcional de fronteras de la UE es transferido a un sitio donde puede tener lugar durante un largo período de tiempo y bajo condiciones en las que la legislación que se aplica no es enteramente la legislación de la UE. Este control extraterritorial todavía se lleva a cabo bajo el control de los Estados miembros, pero lejos de las fronteras comunes o individuales ${ }^{25}$.

${ }_{25}$ MITSILEGAS, Valsamis. Border Security in the European Union: Towards Centralised Controls and Maximum Surveillance. 


\section{El reconocimiento mutuo de la soberanía}

Hay una excepción importante en el Código en cuanto al principio de que todos los nacionales de la lista negra necesitan una visa para entrar en el territorio común. El Código exime a los nacionales de los países en la lista negra de la obligación de obtener una visa si tienen un permiso de residencia válido expedido por un Estado miembro. El artículo 2(15) define un permiso de residencia válido como todos los permisos expedidos por los Estados miembros en el formato común (tratados por el Reglamento 1030/2002, que establece el modelo uniforme de permiso de residencia) $\mathrm{u}$ otros documentos expedidos por los Estados miembros para autorizar la permanencia y el reingreso a su territorio (salvo los solicitantes de asilo en espera de una primera decisión).

El 13 de octubre de 2006, la Comisión publicó la lista de documentos que los Estados miembros le habían notificado en relación con el artículo $2(15)^{26}$. Al momento de la publicación, una serie de Estados miembros aún no habían incluido sus listas, en concreto, Bulgaria, Chipre, Malta y Rumanía (el primero y el último por no haber sido Estados miembros en el momento pertinente). El número de documentos diferentes que un estado expide indica el número de estatus diferentes de residencia que nacionales de terceros países tienen en el territorio. Así, en principio, a mayor cantidad de documentos de residencia que un Estado Miembro emite, más fino éste esculpe las posibles categorías de residencia que tiene en su legislación nacional. Las limitaciones a la residencia de nacionales de terceros países se dividen en tres grupos principales: en primer lugar, la cantidad de tiempo que el individuo puede permanecer en el territorio; esto puede variar de indefinido a muy limitado; en segundo lugar, las actividades que el individuo puede llevar a cabo - por ejemplo, estudios, trabajo, trabajo con un empleador determinado o dentro de una categoría de habilidades, etc; $y$, en tercer lugar, la parte del territorio nacional a la que el individuo debe limitar su residencia.

Los estados necesitan expedir distintos tipos de documentos a los nacionales de terceros países con el fin de establecer el conjunto de derechos a los que tiene acceso el titular. Por lo tanto, a mayor cantidad de tipos de documentos que un estado expide, más preciso ha definido éste qué pueden hacer los diferentes grupos de nacionales de un tercer estado. Por lo tanto, a mayor cantidad de tipos de documentos expide un estado, mayor precisión para definir lo que diferentes grupos de nacionales de terceros países pueden hacer. Así, los números de los diferentes grupos de nacionales de terceros países aumentan con el número de diferentes documentos de residencia que emite el estado. En cualquier caso, a los efectos del cruce de los fronteras exteriores de Schengen, los Estados miembros, en conjunto, han notificado 188 tipos

${ }^{26} 2006 /$ C $247 / 01$. 
diferentes de documentos de residencia que tienen la misma validez que una visa. Esto significa que un guardia de fronteras, en cualquiera de los 1.792 pasos fronterizos exteriores designados, debe ser capaz de identificar: 1. los nacionales de cualquier país que necesiten una visa para entrar en el territorio común; 2. cómo son las visas de Schengen y cuándo son válidas; 3. cuáles son los 188 documentos diferentes emitidos por los Estados miembros que tienen el mismo efecto legal que una visa con el propósito de entrada.

Este es un trabajo especializado que depende de un alto grado de confianza mutua entre los guardias de fronteras de los Estados miembros en cuanto a su capacidad de comprender la ley correctamente. Cuando un guardia de fronteras malinterpreta una ley, el Código establece un derecho de apelación para el individuo al cual se le negó la admisión en la frontera. El guardia de fronteras debe notificar por escrito la razón de la denegación de entrada de acuerdo con el listado de motivos incluidos en el anexo del Código. Él o ella debe proporcionar información sobre la existencia del derecho de apelación y cómo puede ser ejercido, incluso dónde el individuo puede solicitar asistencia jurídica a los efectos de ejercer el derecho de apelación.

\section{Seguridad y autoridad: el uso de la fuerza}

En 2004, la UE adoptó un reglamento que establece la Agencia de Fronteras Exteriores (FRONTEX). Las funciones de la agencia son múltiples, pero entre ellas se encuentra la de dirigir la coordinación operativa entre los Estados miembros en el ámbito de la gestión de las fronteras exteriores ${ }^{27}$. Entre las áreas donde ha habido mucha preocupación en relación a la gestión de la frontera exterior se encuentra España y, en particular, las Islas Canarias. Se pidió a FRONTEX coordinar los controles de los Estados miembros en torno a las Islas Canarias, los cuales fueron diseñados para apoyar a las patrullas fronterizas españolas en la zona. Cada vez que se organizaban estos controles, las operaciones eran designadas con un nombre, siendo el primero de ellos la serie HERA. Para estas operaciones, los Estados miembros fueron invitados a contribuir con embarcaciones y personal guardacostas. Si bien no había ninguna obligación, muchos Estados miembros lo hicieron; no obstante, cada barco guardacostas (y algunos aviones que también se ofrecieron) operaba de acuerdo a sus normas nacionales ${ }^{28}$.

En el 2006 hubo aproximadamente 31.000 cruces fronterizos irregulares a las Islas Canarias en España. Las personas -quienes individualmente representan esos 31.000 cruces- trataban de llegar a España en pequeñas embarcaciones. El

\footnotetext{
27 MITSILEGAS, op. cit.

${ }^{28}$ CARRERA, Sergio. The EU Border Management Strategy: FRONTEX and the Challenges of Irregular Immigration in the Canary Islands.
} 
13 de abril de 2007 se publicó el siguiente comunicado de prensa: “Un número muy bajo de migrantes ilegales [están ahora] Ilegando a las Islas Canarias y más de mil vidas humanas han sido salvadas - ese es el resultado de la operación Hera III coordinado por FRONTEX", dijo el Director Ejecutivo de FRONTEX, Ilkka Laitinen, cuando la operación estaba finalizando ${ }^{29}$.

Según FRONTEX, “(d)urante la operación de dos meses, que comenzó el 12 de febrero, 585 inmigrantes llegaron a las islas, alrededor de un tercio de ellos fueron entrevistados en el marco de la operación. Además, un total de 1167 migrantes fueron desviados a sus puntos de partida en los puertos de la costa de África Occidental, evitando así que arriesgaran sus vidas en un viaje peligroso". La cuestión que se plantea es: itrataban estas personas de entrar en un paso fronterizo designado? Sabemos que la mayoría de estos puntos son los puertos marítimos, que incluyen las Islas Canarias. Si lo hicieran, ifueron tratados de acuerdo con el Código de Fronteras de Schengen y fueron provistos de una decisión por escrito al haberse rechazado su ingreso al territorio junto con las razones y la información con respecto a su derecho de apelación? Si ellas fueron desviadas antes de llegar a un paso fronterizo designado, entonces esto debería haber tenido lugar de conformidad con la legislación española, en la medida en que la desviación se llevó a cabo dentro de las aguas territoriales españolas. Si el desvío tuvo lugar en aguas internacionales, entonces se aplica el derecho internacional marítimo, lo que limita las circunstancias en las que los barcos pueden ser interceptados. Si bien existe una considerable controversia entre los expertos en derecho marítimo sobre este tema (no menos importante debido al interés político de un número de democracias liberales para interceptar barcos con sospecha de extranjeros a bordo antes de que lleguen a las aguas territoriales del estado) la única justificación en la que todos están de acuerdo es el derecho a interceptar si hay vidas en riesgo. El hecho de que FRONTEX utilice el término desviación antes que interceptación se debe a la susceptibilidad que este último, a menos que esté justificado en la legislación internacional, pueda ser equivalente a piratería. Si la desviación se llevó a cabo en las aguas territoriales de un tercer estado, entonces esto tendría que estar en conformidad con las leyes de ese estado. Si las personas en el barco fuesen nacionales de ese estado y estuviesen siendo desviadas de realizar sus actividades (de pesca) normales en aguas territoriales, surge otro problema en relación a la legalidad.

De aquellos que, como resultado de la acción de FRONTEX, fueron incapaces de presentarse en un paso fronterizo designado (las 1,167 personas de quienes el Director habló el 13 de abril de 2007), ¿se hizo un examen

\footnotetext{
${ }^{29}$ Ibidem; JEANDESBOZ, Julien. Reinforcing the Surveillance of EU Borders: The Future Development of FRONTEX and EUROSUR.
} 
individual de su aplicación para arribar a un paso fronterizo designado de Schengen? En caso afirmativo, ¿Sobre qué base fue este examen previo realizado y fueron aplicadas las mismas reglas que se aplicarían bajo el Código Fronterizo Schengen, en particular las relacionadas a la decisión escrita y al derecho de apelación? Estas preguntas pueden parecer algo simples para algunas personas, mal articuladas a una realidad que se muestra en la televisión concerniente a oleadas de migrantes y la inhabilidad de las autoridades estatales de manejarlos como individuos con derecho a una consideración de su aplicación y situación particulares. Sin embargo, estas preguntas derivan de la preocupación central: cómo entender la naturaleza cambiante de las fronteras y su relación con la ley en Europa; qué le sucede al individuo en la tensión entre frontera, territorio y comunidad; y cómo la ley trabaja para proteger a ese individuo.

\section{Conclusiones}

Las demandas de seguridad se mueven en dos direcciones principales, la seguridad colectiva y la seguridad individual. La seguridad del individuo, por ejemplo la seguridad del estatus de residencia, es muy visible y particularizada, mientras que las demandas de seguridad colectiva forman una de las principales maneras en que la seguridad del individuo se desvanece. El individuo como ciudadano o extranjero constituye la piedra angular de la pretensión del sistema europeo de legitimidad: el ciudadano como participante en el gobierno y para cuyo beneficio se lleva a cabo el gobierno. Sin embargo, la comprensión de la brecha entre el ciudadano -el participante esencial en la comunidad (nacional)y el extranjero se ha tornado excepcionalmente compleja en Europa y proviene de una muy amplia gama de fuentes. La relación del ciudadano y el estado se articula en torno a demandas de seguridad. Aunque la propia seguridad como una palabra que expresa algún contenido esencial es muy controvertida, en particular en lo relativo a la forma en que funciona dentro de la relación entre individuo, comunidad y estado, los elementos determinantes son el empoderamiento y la justificación. La proliferación de estatus del individuo entre un ciudadano y un extranjero en Europa puede describirse por su relación con las demandas de exclusión del estado por razones de seguridad (ya sea exclusión territorial, exclusión social en forma de exclusión de la seguridad social $u$ otras modalidades y formas de seguridad) y el empoderamiento a través de la inclusión: convertirse en un miembro del grupo con derecho a reclamar la seguridad como un derecho. La exclusión es la justificación estatal de la seguridad colectiva que entra en conflicto con el reclamo del individuo para su empoderamiento: la seguridad individual.

La tipología de la inclusión y la exclusión europeas, tal como es vista desde la perspectiva de la seguridad y el individuo, puede bosquejarse de la siguiente manera: 
- El ciudadano del estado nacional en Europa: esta es una fuente de seguridad de residencia de la persona a través de la constitución reforzada por el sistema de tratados internacionales, los cuales ya no permiten el destierro o el exilio;

- El ciudadano de la UE que no es nacional del estado donde reside (por ejemplo, ciudadanos polacos viviendo y trabajando en Irlanda): aquí, aunque el individuo es ciudadano de la UE, todavía es pasible de ser expulsado (a Polonia, por ejemplo). El derecho de entrada, residencia, actividad económica y protección contra la expulsión provienen del Tratado de la CE y no están sujetos a cambios por la actuación exclusiva del estado - el estado no es soberano en lo relativo a los ciudadanos de la Unión. La expulsión y la exclusión sólo son posibles por razones de política pública, seguridad pública o salud pública y recae en el estado justificar por qué y cómo la exclusión o expulsión de la persona tiene lugar;

- El ciudadano de la UE que es excluido temporalmente: el caso de los nacionales de Croacia, producto de la ampliación de la UE en 2013; no todos disfrutan del derecho de trabajar en todos los Estados miembros a pesar de que tienen derecho a residir en cualquier parte de la UE; estas restricciones son por un tiempo limitado;

- El nacional suizo (o noruego, islandés, etc.) en un Estado miembro que, si bien no es un ciudadano de la UE y, por lo tanto, es un nacional de un tercer país, disfruta de derechos equivalentes a los de los ciudadanos de la UE;

- El trabajador turco en un país de la UE: sobre la base del acuerdo de 1963 entre la UE y Turquía, los trabajadores turcos también están protegidos al mismo nivel que los ciudadanos de la UE contra la expulsión desde los Estados miembros;

- El nacional de un tercer país que ha vivido legalmente en un Estado miembro durante cinco años o más y, por lo tanto, tiene derecho al estatus de residencia de largo plazo del nacional de terceros países; este nacional de un tercer país goza de una protección contra la expulsión equivalente a los ciudadanos migrantes de la UE;

- El nacional de un tercer país cuyo país de nacionalidad está incluido en la lista blanca de visas de la UE (por ejemplo, nacionales de Estados Unidos): sólo se lo excluye obligatoriamente del territorio de la UE sobre la base de un acto positivo de un Estado miembro para incluir su identidad en una base de datos de personas a ser excluidas por motivos de seguridad; no existe un sistema para evitar que el individuo llegue a la UE, sólo es posible rechazar la admisión; 
- El nacional del tercer país incluido en la lista negra de visas de la UE, pero con el que la UE tiene un acuerdo de facilitación de visas en vigor (por ejemplo, nacionales rusos): aquí el individuo se describe colectivamente como un potencial riesgo para la seguridad de algún tipo (inmigración ilegal, delincuencia, violencia política en función del estado al que pertenece el nacional), pero esta presunción de riesgo de seguridad es parcialmente neutralizada por el acuerdo de facilitación de visas que provee la emisión fácil, rápida y barata de visas para estancias de corta duración en la UE. El lugar donde el control de seguridad se lleva a cabo es el consulado del Estado miembro donde se presenta la solicitud. La certeza de seguridad de la UE, que "compensa" la facilitación de visas, es el cierre de un acuerdo de readmisión a través del cual el estado con acuerdo de facilitación de visas recibe a sus ciudadanos cuando estos son expulsados de la UE (y por lo general también se comprometen a recibir a no nacionales que hayan llegado a la UE a través de dicho estado) ${ }^{30}$;

- El nacional de un tercer país incluido en la lista negra de visas de la UE y con el cual no hay acuerdo de facilitación de visas (por ejemplo, nacionales chinos): existe la presunción de que estas personas representan un riesgo para la seguridad y, por lo tanto, deben obtener una visa en su país de origen en un consulado de un Estado miembro de la $U E$, demostrando que no constituyen un riesgo para la seguridad antes de que se les expida una visa que les permita viajar a la UE;

- El nacional de un tercer país incluido en la lista negra de visados de la UE y cuyo país ha sido especificado por al menos un Estado miembro en el régimen de visas de la UE como un país de preocupación específica de seguridad ${ }^{31}$ : en este caso, la presunción de que el individuo pueda ser una amenaza para la seguridad es mayor que para los nacionales de terceros países cuyos países figuran en la lista negra de visas, pero no en la lista de amenazas a la seguridad.

Los diferentes estatus y su variedad pueden ser entendidos como expresiones de una relación de seguridad entre los individuos, las comunidades y los estados. Las diversas formas de seguridad proporcionan un principio de organización en torno al cual se perfilan la inclusión y la exclusión, tanto territorial como social. La multiplicidad de significados de la seguridad y su contestación puede ser leída a través de las prácticas. La proliferación de las prácticas de semi-inclusión, asignación de derechos y posibilidades indica que

\footnotetext{
30 TRAUNER, Florian, KRUSE, Imke. EC Visa Facilitation and Readmission Agreements: Implementing a New EU Security Approach in the Neighbourhood.

${ }^{31}$ BIGO, GUILD, op. cit.
} 
ya no hay ninguna línea clara y sencilla entre el ciudadano y el extranjero ni líneas de fronteras soberanas en Europa. La división del modo en que la comunidad puede ser descrita a través de sus miembros encuentra sus fuentes y expresiones en una multiplicidad de lugares -leyes nacionales, leyes de la UE, incluyendo leyes internacionales de Derechos Humanos- que están mejor caracterizadas por su diversidad y por la opacidad de sus significados, efectos y justificaciones.

La relación entre la soberanía, la seguridad, las fronteras y las personas está experimentando un cambio sustancial en la UE. Hay una transformación fundamental que tiene lugar con respecto a la posición de autoridad en la UE y las consecuencias de esa autoridad sobre los individuos. Mientras que los uniformes de los funcionarios que están llevando a cabo controles fronterizos no han cambiado, lo que hacen y dónde lo hacen sí lo ha hecho. El estado de derecho, un principio profundamente arraigado en los Estados miembros, exige una nueva jerarquía respecto a cómo el orden nacional se articula para cumplir con los requisitos de la legislación de la UE.

La soberanía, en forma de autoridad, está migrando lejos de los límites territoriales del estado y, en cambio, se la puede encontrar operando muy adentro del territorio de otros estados a través de normas de visa, en alta mar y en otros lugares. El argumento político para la migración de la soberanía es la seguridad, sin embargo, la seguridad de quién y en nombre de quién se torna más opaca a medida que el ejercicio de la autoridad se aleja de su hogar territorial tradicional.

Dos ideas clave orientan mi pensamiento. En primer lugar, la sociología política internacional: seguir al individuo más que al estado para comprender el significado de la migración para los estados, la comunidad internacional y los propios migrantes. A través de este enfoque crítico de los estudios de migración uno puede revelar cómo se estructura y utiliza el poder estatal y dónde se encuentran y cómo operan las posibilidades de resistencia para el individuo. En segundo lugar, la relación entre la seguridad y la inseguridad como un continuum que permite que la inseguridad siga al sujeto -el extranjero, el inmigrante- a pesar de que los individuos son completamente diferentes, tienen trayectorias y objetivos distintos. Mi propósito aquí ha sido unir ambos marcos desafiando la noción central de los estudios migratorios: la frontera entre los estados. Al hacerlo, he seguido al individuo en movimiento, desafiando y resistiendo la categorización del Estado como un extranjero y como una cuenta más en el continuum del collar de la inseguridad. 


\section{Referencias bibliográficas}

ALBERT, Mathias; JACOBSON, David; LAPID, Yosef. Identities, Borders, Orders: Rethinking International Relations Theory. Minneapolis, MN: University of Minnesota Press, 2001.

ATCER, Anaïs Faure. The Abolition of Internal Border Checks in an Enlarged Schengen Area: Freedom of movement or a scattered web of security checks?. CEPS Challenge Liberty and Security Research Paper n. 8 (marzo). Bruselas: Centre for European Policy Studies, 2008.

BALDACCINI, Anneliese; GUILD, Elspeth; TONER, Helen. Whose Freedom, Security and Justice?: EU Immigration and Asylum Law and Policy. Londres: Bloomsbury Publishing, 2007.

BALZACQ, Thierry; CARRERA, Sergio. Migration, Borders and Asylum: Trends and Vulnerabilities in EU Policy. Bruselas: Centre for European Policy Studies, 2005.

BIGO, Didier; GUILD, Elspeth (eds.). Controlling Frontiers: Free Movement Into and Within Europe. Hampshire: Ashgate, 2003.

BROUWER, Evelien. Digital Borders and Real Rights: Effective Remedies for ThirdCountry Nationals in the Schengen Information System. Leiden: Brill, 2008.

CARBY-HALL, Jo. The Treatment of Polish and A8 Economic Migrants in the European Union Member States. Warsaw: Bureau of the Commissioner for Civil Rights Protection, 2008.

CARRERA, Sergio. The EU Border Management Strategy: FRONTEX and the Challenges of Irregular Immigration in the Canary Islands. CEPS Working Documents, n. 261. Bruselas: Centre for European Policy Studies, 2007.

ELSEN, Charles. Schengen et la cooperation dans les domains de la justice et des affaires interiors. Besoins actuels et options futures. In DEN BOER, Monica (ed.). The Implementation of Schengen: First the Widening, Now the Deepening. Maastricht: EIPA, 1997.

GORTÁZAR, Cristina. Spain: Two Immigration Acts at the End of the Millennium. European Journal of Migration and Law, v. 4, n. 1, 2002, p. 1-21.

GUILD, Elspeth. Moving the Borders of Europe. Lovaina: Katholieke University, 2001.

GUILD, Elspeth. The Uses and Abuses of Counter-Terrorism Policies in Europe: The Case of the 'Terrorist Lists'. JCMS: Journal of Common Market Studies, v. 46, n. 1, 2008, p. 173-193.

JEANDESBOZ, Julien. Reinforcing the Surveillance of EU Borders: The Future Development of FRONTEX and EUROSUR. CEPS Challenge Liberty and Security Research Paper, n. 11 (Agosto). Bruselas: Centre for European Policy Studies, 2008.

JORRY, Hélène. Construction of a European Institutional Model for Managing Operational Cooperation at the EU's External Borders: Is the FRONTEX Agency a decisive step forward?. CEPS Challenge Liberty and Security Research Paper, $\mathrm{n}$. 6 (marzo). Bruselas: Centre for European Policy Studies, 2007. 
MELONI, Annalisa. Visa Policy Within the European Union Structure. Berlin: Springer, 2006.

MITSILEGAS, Valsamis. Border Security in the European Union: Towards Centralised Controls and Maximum Surveillance. Oxford: Hart Publishing, 2007.

POTEMKINA, Olga; KAVESHNIKOV, Nikolay. Russia and the European Union: 'Cold Summer'. Modern Europe, n. 3, 2007, p. 24-39.

TRAUNER, Florian; KRUSE, Imke. EC Visa Facilitation and Readmission Agreements: Implementing a New EU Security Approach in the Neighbourhood. CEPS Working Document, n. 290. Bruselas: Centre for European Policy Studies, 2008. TRIDIMAS, Takis. The General Principles of EU Law. Oxford: Oxford University Press, 2007.

WEBER, Max. The Fundamental Concepts of Sociology. In The Theory of Social and Economic Organization. Londres: Collier Macmillan, 1964.

Recibido para publicación en 01.11.2017

Aceptado para publicación en 23.11.2017

Received for publication in November 015t, 2017

Accepted for publication in November 23 ${ }^{\text {th }}, 2017$

ISSN impresso 1980-8585

ISSN eletrônico 2237-9843

http://dx.doi.org/10.1590/1980-85852503880005108 